\title{
Adjusted Forms of the Fourier Coefficient Asymptotic Expansion and Applications in Numerical Quadrature*
}

\author{
By J. N. Lyness
}

\begin{abstract}
The conventional Fourier coefficient asymptotic expansion is derived by means of a specific contour integration. An adjusted expansion is obtained by deforming this contour. A corresponding adjustment to the Euler-Maclaurin expansion exists. The effect of this adjustment in the error functional for a general quadrature rule is investigated. It is the same as the effect of subtracting out a pair of complex poles from the integrand, using an unconventional subtraction function. In certain applications, the use of this subtraction function is of practical value.

An incidental result is a direct proof of Erdélyi's formula for the Fourier coefficient asymptotic expansion, valid when $f(x)$ has algebraic or logarithmic singularities, but is otherwise analytic.
\end{abstract}

1. The Fourier Coefficient Asymptotic Expansion. The Fourier coefficient asymptotic expansion (F.C.A.E.) (1.3) below is a classical formula which is elementary to derive using a standard application of the formula for integration by parts, namely,

$$
\int_{a}^{b} f(x) e^{i k x} d x=\frac{e^{i k b} f(b)-e^{i k a} f(a)}{i k}-\frac{1}{i k} \int_{a}^{b} f^{\prime}(x) e^{i k x} d x .
$$

The integral on the right is almost the same as that on the left; the only difference is that $f^{\prime}(x)$ replaces $f(x)$. Consequently, the formula may be applied iteratively. So long as

$$
f(x) \in C^{(p)}[a, b]
$$

this leads to the following series:

$$
\begin{aligned}
\int_{a}^{b} f(x) e^{i k x} d x= & -e^{i k b}\left\{\frac{i}{k} f(b)+\frac{i^{2}}{k^{2}} f^{\prime}(b)+\cdots+\frac{i^{p}}{k^{p}} f^{(p-1)}(b)\right\} \\
& +e^{i k a}\left\{\frac{i}{k} f(a)+\frac{i^{2}}{k^{2}} f^{\prime}(a)+\cdots+\frac{i^{p}}{k^{p}} f^{(p-1)}(a)\right\} \\
& +\frac{i^{p}}{k^{p}} \int_{a}^{b} f^{(p)}(x) e^{i k x} d x .
\end{aligned}
$$

This series is equally familiar in the form it takes for a Fourier coefficient. If we set $a=0, b=1, k=2 \pi m$, collect the final terms in the series in the remainder, and separate real and imaginary parts, we find

$$
2 C^{(m)} f=2 \int_{0}^{1} f(x) \cos 2 \pi m x d x=\sum_{a=1}^{[(p-1) / 2]} \frac{K_{2 q}}{m^{2 q}}+2 C_{p}^{(m)} f,
$$

Received February 5, 1970, revised July 8, 1970.

AMS 1969 subject classifications. Primary 4210, 6555; Secondary 4208.

Key words and phrases. Fourier coefficients, Euler-Maclaurin summation formula, Fourier coefficient asymptotic expansion, numerical quadrature, subtracting out singularities.

* Work performed under the auspices of the U. S. Atomic Energy Commission. 


$$
2 S^{(m)} f=2 \int_{0}^{1} f(x) \sin 2 \pi m x d x=\sum_{q=0}^{[(p-2) / 2\}} \frac{K_{2 q+1}}{m^{2 q+1}}+2 S_{p}^{(m)} f,
$$

where

$$
\begin{aligned}
K_{2 q} & =2(-1)^{a-1}\left(f^{(2 q-1)}(1)-f^{(2 q-1)}(0)\right) /(2 \pi)^{2 q}, \\
K_{2 q+1} & =2(-1)^{q-1}\left(f^{(2 q)}(1)-f^{(2 q)}(0)\right) /(2 \pi)^{2 q+1},
\end{aligned}
$$

and the remainder terms $2 C_{p}^{(m)} f$ and $2 S_{p}^{(m)} f$ are of order $m^{-\nu}$ for large $m$.

By allowing $p$ to become infinite in series such as (1.3), (1.4), and (1.5), we obtain expansions that we refer to as Fourier coefficient asymptotic expansions. When $f(x)$ is a specified polynomial, these expansions terminate, leaving a closed expression for the trigonometric integral. More generally, when $f(x)$ is a function which is simple to differentiate, these expansions appear at first sight to provide a reasonable basis for the numerical evaluation of the integral. In practice, this appearance is most deceptive. In Lyness [4, Section 4], several illustrations are given in which the most inconvenient behavior is manifest. Perhaps the most flamboyant example is

$$
f(x)=e^{\alpha x}+e^{\cos 2 \pi x}, \quad|\alpha|<m,
$$

for which the expansion converges to a result different from $2 C^{(m)} f$. However, the F.C.A.E. is generally divergent. For large values of $m$, the early terms in the series have the numerical appearance of a convergent series, but later terms increase in magnitude. Here again, the situation may be deceptive. In the example with $m=6$ and

$$
f(x)=1 /\left(x^{2}-x+0.26\right),
$$

the magnitude of the terms in (1.4) initially decreases but then increases. The smallest term is the eighth, which is about $-10^{-9}$. Terminating the series after the $q$ th term, $5 \leqq q \leqq 15$, results in an approximation of $-2.023 \times 10^{-2}$. The true value of $C^{(6)} f$ is +0.701 .

It is hardly surprising that an asymptotic expansion having such disconcerting numerical properties is rarely used for numerical calculation. One of the annoying aspects of the situation is that the elementary derivation of the series given above does not indicate at all clearly the source of this "unreliability." In Section 2 we give a different proof of (1.3) based on contour integration. This proof is valid when $f(x)$ is an analytic function that has an analytic continuation $f(z)$ regular in a region $R$ of the complex plane which contains the interval of integration. The proof relates the remainder term to the analytic properties of $f(z)$ in a manner in which the principal contributions to the remainder term can be recognized. In very simple cases an important part of this "error" can be "subtracted out".

The series (1.3) exists so long as $f(x)$ satisfies restriction (1.2), i.e., has continuous derivatives of orders zero through $p$. However, if $f(x)$ fails to satisfy (1.2), Eq. (1.3) is not generally valid. If $f(x)$ has a finite number of algebraic singularities on an interval but otherwise has continuous derivatives of orders zero through $p$, a different analogous series exists. This series may be constructed by treating each interval between adjacent singularities separately and combining the result. Between two such singularities, located for convenience at $a$ and $b$, the function $f(x)$ takes the form

$$
f(x)=(x-a)^{\alpha}(b-x)^{\beta} h(x), \quad \alpha, \beta>-1,
$$


where $\alpha$ and $\beta$ are not in general integers, and

$$
h(x) \in C^{(p)}[a, b] .
$$

A result that corresponds to (1.3) was given by Erdélyi [1] in 1954. The derivatives of $f(x)$ at $a$ and $b$ are replaced by derivatives of $\psi(x)$ and $\phi(x)$, the 'regular parts' of $f(x)$ at $a$ and at $b$, respectively. These functions are defined by

$$
\psi(x)=(b-x)^{\beta} h(x), \quad \phi(x)=(x-a)^{\alpha} h(x) .
$$

The coefficients in (1.3) and the indices are also adjusted. The resulting asymptotic expansion is as follows:

THEOREM 1.12. If $f(x)$ is given by (1.9), $\psi(x)$ and $\phi(x)$ are defined by (1.10), and if

$$
h(x) \in A[a, b]
$$

then for $k>0$

$$
\begin{aligned}
\int_{a}^{b} f(x) e^{i k x} d x \simeq & -e^{i k b-i \pi \beta / 2} \sum_{a=0}^{p^{2}-1} \frac{\phi^{(a)}(b) i^{q+1}(q+\beta) !}{k^{a+\beta+1} q !} \\
& +e^{i k a+i \pi \alpha / 2} \sum_{a=0}^{p_{2}-1} \frac{\psi^{(a)}(a) i^{q+1}(q+\alpha) !}{k^{q+\alpha+1} q !} \\
& +O\left(k^{-\left(p_{1}+\beta+1\right)}\right)+O\left(k^{-\left(p_{2}+\beta+1\right)}\right) \quad \text { ask } \rightarrow \infty,
\end{aligned}
$$

for

$$
p_{1}, p_{2} \geqq 0 \text {. }
$$

This result reduces to (1.3) when $\alpha$ and $\beta$ are either zero or integers.

The theorem just stated requires that $h(x)$ be analytic in a region $R$ that contains the interval $[a, b]$. In fact, a stronger form of this theorem is valid.

THEOREM 1.13. Theorem 1.12 is valid if restriction (1.11) is replaced by

$$
h(x) \in C^{(p)}[a, b],
$$

under the additional restriction

$$
p_{1}, p_{2} \leqq p
$$

Naturally, Theorem 1.12 is a direct corollary of Theorem 1.13.

The author has seen two proofs of this theorem. The first, in 1954 (Erdélyi [1]), generalizes the technique of integration by parts (1.1) making use of neutralizer functions. The second (Lighthill [2]) uses the theory of generalized functions. Unless the reader is familiar with these concepts, it is difficult to acquire from the proofs any idea about the magnitude of the remainder term, apart, of course, from its order.

For readers interested in the specific case in which $f(x)$ is an analytic function, Theorem 1.12 is sufficient. In this case, a proof can be based on generally more familiar concepts. The result is obtained by use of contour integration and the remainder term appears as the sum of specific contour integrals of well-defined functions. Besides showing the order (for large $k$ ) of the remainder term, the proof gives at least a rough idea of its magnitude for specified finite values of $k$.

The proof and the theorem may be adjusted to cover the case in which $f(x)$ has a logarithmic singularity. This is described in the Appendix. 
2. Proof of Theorem 1.12. In this section, we prove Theorem 1.12. Here $f(x)$, $h(x), \psi(x)$, and $\phi(x)$ are real functions of a real variable, defined as in (1.9) and (1.10). In addition, each has an analytic continuation into the complex plane. The function $h(z)$ is regular (has no singularities) within a region $Q$ that contains the rectangle with vertices $a \pm i L, b \pm i L$ where $L$ is a positive constant. If the singularities of $h(z)$ lie at $\lambda_{1} \pm i \mu_{i}$, clearly,

$$
L<M=\min _{a \leqq \lambda_{i} \leq b}\left|\mu_{i}\right| .
$$

For convenience we subdivide the proof into four lemmas. The proof of each lemma is straightforward.

LEMMA 2.1.

$$
\int_{a}^{b} f(x) e^{i k x} d x=\int_{a}^{a+i L} f(z) e^{i k z} d z+\int_{b+i L}^{b} f(z) e^{i k z} d z+O\left(e^{-k L}\right) \text { as } k \rightarrow \infty .
$$

(Unless stated otherwise, all integrals in this paper are along straight-line contours.)

Since $f(z) e^{i k z}$ is regular within the rectangle mentioned above, the contour between $a$ and $b$ may be deformed and the integral replaced by the sum of three integrals, connecting successively $a, a+i L, b+i L, b$. The right-hand side of (2.2) includes two of these explicitly. To establish Lemma 2.1, we have to show that the third integral, that which connects $a+i L$ and $b+i L$, is of the stated order $O\left(e^{-k L}\right)$.

We define

$$
N_{L}=\max _{a \leq x \leqq b}|f(x+i L)| .
$$

Then, using elementary inequalities, we have

$$
\left|\int_{a+i L}^{b+i L} f(z) e^{i k z} d z\right|=\left|e^{-k L} \int_{a}^{b} f(x+i L) e^{i k x} i d x\right|<(b-a) N_{L} e^{-k L} .
$$

This establishes Lemma 2.1.

The next lemma deals with the effect of replacing $f(z)$ in the neighborhood of $a$ by an approximation $g(z)$ based on the first $p$ terms of the Taylor expansion of the regular part of $f(z)$. We define $g(z)$ as

$$
g(z)=(z-a)^{\alpha}\left(\psi(a)+(z-a) \psi^{\prime}(a)+\cdots+\frac{(z-a)^{p-1}}{(p-1) !} \psi^{(p-1)}(a)\right) .
$$

LEMMA 2.2.

$$
\int_{a}^{a+i L} f(z) e^{i k z} d z=\int_{a}^{a+i L} g(z) e^{i k z} d z+O\left(k^{-\alpha-p-1}\right) .
$$

An elementary application of the mean value theorem indicates that at all points $z=a+i y$, the value of $\psi(z)$ differs from the sum of the first $p$ terms in its Taylor expansion by an amount whose modulus is less than $y^{p} \psi^{(p)}(a+i \xi) / p$ ! where $0 \leqq$ $\xi \leqq y$. Consequently, the function

$$
r(z)=f(z)-g(z)
$$

satisfies the inequality

$$
|r(a+i y)| \leqq|y|^{p+\alpha} M_{p} / p !, \quad 0<y<L,
$$


where

$$
M_{p}=\max _{0 \leq \theta \leq 1}\left|\psi^{(p)}(a+i \theta L)\right|
$$

Thus,

$$
\begin{aligned}
\left|\int_{a}^{a+i L} r(z) e^{i k z} d z\right| & =\left|e^{i k a} \int_{0}^{L} r(a+i y) e^{-k y} i d y\right| \\
& \leqq \frac{M_{p}}{p !} \int_{0}^{L} y^{p+\alpha} e^{-k y} d y \leqq \frac{M_{p}}{p !} \int_{0}^{\infty} y^{p+\alpha} e^{-k y} d y \\
& =\frac{M_{p}}{p ! k^{\alpha+p+1}} \int_{0}^{\infty} t^{p+\alpha} e^{-t} d t=\frac{M_{p}(p+\alpha) !}{p ! k^{\alpha+p+1}}
\end{aligned}
$$

This establishes Lemma 2.2.

Next, we proceed to the evaluation of the integral on the right-hand side of (2.6). We now show

LEMMA 2.3.

$$
\int_{a}^{a+i L} g(z) e^{i k z} d z=\int_{a}^{a+i \infty} g(z) e^{i k z} d z+O\left(k^{-1} e^{-k L}\right) .
$$

In view of the definition (2.5) of $g(z)$, the difference between these two integrals may be expressed as

$$
\int_{a+i L}^{a+i \infty} g(z) e^{i k z} d z=\sum_{a=0}^{p-1} \frac{\psi^{(a)}(a)}{q !} \int_{a+i L}^{a+i \infty}(z-a)^{a+\alpha} e^{i k z} d z
$$

Each of the integrals on the right-hand side may be expressed in terms of the incomplete gamma function $\Gamma(\beta+1, x)$, defined by

$$
\Gamma(\beta+1, x)=\int_{x}^{\infty} t^{\beta} e^{-t} d t
$$

Before doing this, we establish the order of $\Gamma(\beta+1, x)$ for large $x$. When $-1<\beta \leqq 0$, the integrand in (2.13) is less than or equal to $x^{\beta} e^{-t}$. Thus

$$
\Gamma(\beta+1, x)<x^{\beta} \int_{x}^{\infty} e^{-t} d t=x^{\beta} e^{-x},
$$

so that

$$
\Gamma(\beta+1, x) \sim O\left(x^{\beta} e^{-x}\right), \quad-1<\beta \leqq 0 .
$$

For positive values of $\beta$, integration by parts of the integral in (2.13) leads to

$$
\Gamma(\beta+1, x)=x^{\beta} e^{-x}+\beta \Gamma(\beta, x) .
$$

In view of (2.15), it follows that

$$
\Gamma(\beta+1, x) \sim O\left(x^{\beta} e^{-x}\right), \quad \beta>-1 .
$$

If we set $z=a+i y$ and $t=k y$ and use (2.13), expression (2.12) may now be manipulated as follows:

$$
\begin{aligned}
\int_{a+i L}^{a+i \infty} g(z) e^{i k z} d z & =\sum_{a=0}^{p-1} \frac{\psi^{(a)}(a)}{q !} e^{i \pi \alpha / 2} e^{i k a} i^{a+1} \int_{L}^{\infty} y^{Q+\alpha} e^{-k y} d y \\
& =e^{i \pi \alpha / 2} e^{i k a} \sum_{a=0}^{p-1} \frac{\psi^{(a)}(a)}{q !} \frac{i^{a+1}}{k^{\alpha+a+1}} \Gamma(\alpha+q+1, k L) .
\end{aligned}
$$


Since the incomplete gamma function is of order $O\left((k L)^{\alpha+a} e^{-k L}\right)$, the sum in (2.18) contains $p$ terms, each of order $k^{-1} e^{-k L}$, and so is itself of order $k^{-1} e^{-k L}$. This establishes Lemma 2.3. Finally, we require

LEMMA 2.4 .

$$
\int_{a}^{a+i \infty} g(z) e^{i k z} d z=e^{i \pi \alpha / 2} e^{i k a} \sum_{a=0}^{p-1} \frac{\psi^{(a)}(a)}{q !} \frac{i^{q+1}}{k^{\alpha+q+1}} \Gamma(\alpha+q+1) .
$$

This lemma follows from (2.18) by setting $L=0$ and noting from (2.13) that

$$
\Gamma(\alpha+q+1,0)=\Gamma(\alpha+q+1) .
$$

Proof of Theorem 1.12. The terms of orders $O\left(e^{-k L}\right), O\left(k^{-\alpha-p-1}\right)$ and $O\left(k^{-1} e^{-k L}\right)$ that occur in Lemmas 2.1, 2.2, and 2.3 are each of proper order to appear in the remainder term in (1.12).

Lemmas 2.2, 2.3, and 2.4 successively reduce the first integral on the right in Lemma 2.1 to the terms in (1.12) involving derivatives of $\psi(x)$ at $x=a$, together with contributions to the remainder term. A similar treatment of the second integral on the right in Lemma 2.1 leads to the other term in (1.12) involving derivatives of $\phi(x)$ at $x=b$ and other proper contributions to the remainder term. This establishes Theorem 1.12 .

We close with two comments on the proof. First, the use of the incomplete gamma function is a notational convenience only. All we require is that the integral in (2.13) be of order $x^{\beta} e^{-x}$ for $\beta>-1$. No other properties are required. Secondly, the remainder term as derived here includes terms of order $O\left(e^{-k L}\right)$ and $O\left(k^{-1} e^{-k L}\right)$. Since, by (2.1), $L$ may take any value less than $M$, the entire proof may be repeated with $L^{\prime}=(L+M) / 2$ instead of $L$ to yield terms of order $O\left(e^{-k L^{\prime}}\right)$ and $O\left(k^{-1} e^{-k L^{\prime}}\right)$. Thus, these remainder terms are in fact $o\left(e^{-k L}\right)$ and $o\left(k^{-1} e^{-k L}\right)$. However, the principal part of the remainder term is generally either $O\left(k^{\alpha+p_{1}+1}\right)$ or $O\left(k^{\alpha+p_{2}+1}\right)$, as stated

3. The Remainder Term. In this section we discuss the Fourier coefficient asymptotic expansion with a view to its possible application with finite $k$. To this end, we take a closer look at some of the features of the proof just presented. In the proof, the integral of $f(x) e^{i k x}$ along the real axis is replaced by a contour integral of $f(z) e^{i k z}$. The contour is arranged in such a way that, except for the terminal points $a$ and $b$, it lies in the upper half-plane At any point $x+i y$ on this contour, the contribution to the integral is proportional to

$$
f(x+i y) e^{i k x} e^{-k y} \text {. }
$$

For all points, except $a$ and $b, y$ is positive and (3.1) is of order $e^{-k y}$ and so may be relegated to the remainder term. To obtain the terms in the series, one considers only the parts of the contour immediately adjacent to $a$ and to $b$.

The first three lemmas may be restated as follows:

LEMMA 3.1.

$$
\int_{a+i L}^{b+i L} f(z) e^{i k z} d z=I_{1} \sim O\left(e^{-k L}\right)
$$

LEMMA 3.2 .

$$
\int_{a}^{a+i L} r(z) e^{i k z} d z=I_{2} \sim O\left(k^{-\alpha-p-1}\right)
$$


LEMMA 3.3.

$$
\int_{a+i L}^{a+i \infty} g(z) e^{i k z} d z=I_{3} \sim O\left(k^{-1} e^{-k L}\right)
$$

Theorem 1.12 follows because the remainder term is equal to $I_{1}+I_{2}+I_{3}+$ $I_{4}+I_{5}$, and all these contributions are of appropriate order. $\left(I_{4}\right.$ and $I_{5}$ correspond to $I_{2}$ and $I_{3}$ and arise from the other terminal point $b$.)

For finite values of $k$, it is the magnitude rather than the order of these contributions that is significant. Obviously, it is not feasible to evaluate these integrals. And the situation is further complicated by the fact that contours can be deformed, leading to different but analogous expressions. Contour deformations do not alter the value of the remainder term, but do redistribute the contributions among the components.

Rather than continue this discussion in general terms, we look at the two examples (1.7) and (1.8) mentioned in Section 1. In the case of (1.8), $f(z)$ has a pole at $z=$ $0.5+(0.1) i$. Consequently, $I_{1}$ involves an integral whose contour passes within a distance 0.1 of the real axis. It follows that, at some point on this contour, $|f(z)|>$ $f(0.5)=100$. From (2.4), we see that we have estimated $I_{1}$ using $I_{1}<N_{L} e^{-k L}$, where $N_{L}>100$. The numerical results mentioned after (1.8) refer to the case with $m=6$. With $k=12 \pi, L<0.1$ and $N_{L}>100$, this estimate gives very roughly that $\left|I_{1}\right| \sim$ $100 e^{-1.2 \pi} \simeq 2.5$. This estimate is very poor. Actually, $I_{1}$ as defined in (3.1) is about 0.7 . The true value of the Fourier coefficient is also about 0.7 . The relegation of the term $I_{1}$ to the remainder leads to an estimate of -0.02 instead of 0.7 for the Fourier coefficient.

In example (1.7), it is the contribution of the function $e^{\cos 2 \pi x}$ which causes the numerical difficulty. For this function, $I_{2}+I_{3}+I_{4}+I_{5}=0$ and $I_{1}$ is identical with the original integral these being of order $O\left(e^{-k L}\right)$. To treat $I_{1}$ as a remainder term renders the calculation meaningless.

In other examples investigated by the author, it is the neglect of the contribution from $I_{1}$-or the main part of the contour-which accounts mainly for misleading numerical results. This, unfortunately, cannot be rectified by including an additional term in the series, since $I_{1}$ is independent of $p$, the number of included terms. The effect of adding additional terms is to alter the definitions in $I_{2}$ and $I_{3}$, essentially reducing the order of $I_{2}$ from $O\left(k^{-\alpha-p-1}\right)$ to $O\left(k^{-\alpha-p-2}\right)$ and introducing into $I_{3}$ an extra term of the same order $O\left(k^{-1} e^{-k L}\right)$ as the others.

In general, the use of any truncated series for numerical calculation should be undertaken only if evidence is available that the numerical value of the remainder term is smaller than the required tolerance. These examples show that for the truncated F.C.A.E., a knowledge of the order of the remainder term is not sufficient.

The foregoing discussion is set in terms of numerical calculation. In practice, this expansion is rarely used for numerical calculation directly. It is, however, used indirectly. The Euler-Maclaurin summation formula (5.3) below is an asymptotic expansion of the same nature, and may be derived by taking an infinite sum of different Fourier coefficient asymptotic expansions. And the derivation of numerical quadrature rules may be based on the Euler-Maclaurin summation formula. Thus, inconvenient behavior of the type mentioned above occurs also (but in a less critical manner) in numerical quadrature. 
4. The F.C.A.E. Adjusted for a Single Pole. In the rest of this paper we develop a single theme. We suppose that the function $f(z)$ has a pole at $z=c=\lambda \pm i \mu$, where $0<\lambda<1$ : $\mu$ may be thought of as being small. In this case, $L$ is small also, and for moderate values of $k$ the expansion is unreliable. However, the proof could have been carried through using a larger value of $L$. The contour would then enclose the pole and an additional contribution, arising from the residue of $f(z) e^{i k z}$ at the pole, would appear in the remainder term. Since the precise value of this term is known, it can be included in the series to yield to an "adjusted" Fourier coefficient asymptotic expansion.

In this section we derive this expansion. In the subsequent section we derive the corresponding "adjusted" Euler-Maclaurin expansion. Then, in the final section, we note the effect of this adjustment in the error functional for quadrature rules. It corresponds there to an unconventional method of subtracting out complex singularities.

The behavior of this term is independent of whether or not $f(z)$ has algebraic singularities at the end points $a$ and $b$. It is convenient from this point on to consider only the regular case for the unit interval. We set

$$
a=0 ; \quad b=1 ; \quad \alpha=\beta=0 ; \quad k=2 \pi m ; \quad \psi(x)=\phi(x)=f(x) .
$$

In this section, we use both $k$ and $2 \pi m$ as may be notationally convenient. The results apply in trivially modified form to the more general case.

The configuration is then as follows. The function $f(z)$ is regular in a region $R$ that contains the rectangle with vertices $\pm i L, 1 \pm i L$. There are poles of order $\omega$ at $z=c$ and at $z=\bar{c}$, where

$$
c=\lambda+i \mu, \quad 0<\lambda<1, \quad \mu>0 .
$$

With the exception of these poles, $f(z)$ is regular in a larger region, which contains the rectangle having vertices $\pm i L^{\prime}, 1 \pm i L^{\prime}$. There is a further singularity at

$$
z=C^{\prime}=\Lambda^{\prime}+i M^{\prime}, \quad 0<\Lambda^{\prime}<1, \quad M^{\prime}>0 .
$$

Apart from the poles at $z=c$ and at $z=\bar{c}, C^{\prime}$ is the nearest singularity to the real axis having a real part in the interval $[0,1]$. We take $L^{\prime}>\mu$. Thus

$$
0<L<M=\mu<L^{\prime}<M^{\prime} \text {. }
$$

The derivation of the expansion in Section 2 gives a specific representation for the remainder term as a sum of contour integrals. This remainder term coincides with $C_{p}^{(m)} f+i S_{p}^{(m)} f$ introduced in (1.3) and (1.4).

In view of (4.1), we have

$$
f(z)=g(z)+r(z)=h(z)+s(z),
$$

where

$$
\begin{aligned}
& g(z)=\sum_{a=0}^{p-1} f^{(a)}(0) z^{Q} / q ! \\
& h(z)=\sum_{a=0}^{p-1} f^{(a)}(1)(z-1)^{q} / q ! .
\end{aligned}
$$


We define $C_{p}^{(m)}(L) f$ and $S_{p}^{(m)}(L) f$ as real numbers by

$$
\begin{aligned}
C_{p}^{(m)}(L) f+i S_{p}^{(m)}(L) f & =\int_{i \infty}^{i L} g(z) e^{i k z} d z+\int_{0}^{i L} r(z) e^{i k z} d z \\
& +\int_{i L}^{1+i L} f(z) e^{i k z} d z+\int_{1+i L}^{0} s(z) e^{i k z} d z+\int_{1+i L}^{1+i \infty} h(z) e^{i k z} d z,
\end{aligned}
$$

and then it follows from Section 2 that

$$
C_{p}^{(m)} f+i S_{p}^{(m)} f=C_{p}^{(m)}(L) f+i S_{p}^{(m)}(L) f, \quad 0<L<M .
$$

The function of $L$ defined by (4.8) takes the same value for all values of $L$ in the interval $(0, M)$, though, of course, the individual components on the right-hand side are generally different for different values of $L$. In fact, it is elementary to verify from (4.8) and (4.5) that

$$
C_{p}^{(m)}\left(L_{2}\right) f+i S_{p}^{(m)}\left(L_{2}\right) f-C_{p}^{(m)}\left(L_{1}\right) f-i S_{p}^{(m)}\left(L_{1}\right) f=\oint_{R} f(z) e^{i k z} d z,
$$

the contour $R$ being the rectangle connecting the points $i L_{1}, i L_{2}, 1+i L_{2}, 1+i L_{1}, i L_{1}$ in that order, or, of course, any allowable deformation of this contour. If we choose $L_{2}=L$ and $L_{1}=L^{\prime}$, where $L$ and $L^{\prime}$ satisfy inequalities (4.4), the closed contour integral includes the pole at $z=c$. In view of (4.10), we find that

$$
C_{p}^{(m)} f+i S_{p}^{(m)} f=C_{p}^{(m)}\left(L^{\prime}\right) f+i S_{p}^{(m)}\left(L^{\prime}\right) f+2 \pi i \operatorname{Res}(2 \pi m),
$$

$$
M<L^{\prime}<M^{\prime} \text {, }
$$

where we have denoted by $\operatorname{Res}(k)$ the residue of $f(z) e^{i k z}$ at the pole at $z=c$.

The value of Res $(k)$ is determined as follows. If $f(z)$ has a pole of order $\omega$ at $z=c$, then there exists an expansion

$$
f(z)=\sum_{r=-\infty}^{\infty} a_{r}(z-c)^{r}
$$

Similarly,

$$
e^{i k z}=e^{i k c} \sum_{s=0}^{\infty}(i k)^{s}(z-c)^{s} / s ! .
$$

Res $(k)$ is the coefficient of $(z-c)^{-1}$ in the product of these expressions, namely,

$$
\operatorname{Res}(k)=e^{i k c} \sum_{r=1}^{\omega} a_{-r}(i k)^{r-1} /(r-1) ! .
$$

If $f(z)$ has a simple pole $(\omega=1)$ and is of the form

$$
f(z)=\theta(z) /(z-c)(z-\bar{c})
$$

then

$$
\operatorname{Res}(k)=a_{-1} e^{i k c}=\theta(c) e^{i k c} / 2 i \mu .
$$

We intend to adjust the asymptotic expansion by taking the term $2 \pi i$ Res $(2 \pi m)$ out of the remainder term and treating it as part of the expansion. It must be emphasized, however, that what we are doing is making an adjustment which has the effect 
of removing terms of order $O\left(e^{-k L}\right), L<M$, from the remainder and replacing these by terms of order $O\left(e^{-k L^{\prime}}\right), L^{\prime}<M^{\prime}$, which are ultimately smaller. But the order of the remainder term, which is $O\left(k^{-p}\right)$, remains unaltered. No justification is required for this procedure: in general, the addition of a term $A e^{-B k}$ to this asymptotic expansion does not affect the expansion's validity. Our motivation for adding the particular term $2 \pi i \operatorname{Res}(2 \pi m)$ is simply that it appears that for finite $k$, the numerical value of the remainder will be reduced. Thus we conclude this section with a definition rather than a theorem. In fact, we need two definitions.

Definition 4.17. A Fourier coefficient asymptotic expansion is denoted as standard if the order of the remainder term is higher than the order of any terms occurring in the expansion.

Expansions (1.12), (1.4), and (1.5) are standard; expansion (1.3) fails to be standard, but would be if the final terms were taken into the remainder term.

Definition 4.18. A Fourier coefficient asymptotic expansion is said to be adjusted for a pole of $f(z)$ at $z=c$ if a single term $2 \pi i$ Res $(k)$ is added into a standard expansion for $\int_{a}^{b} f(x) e^{i k x} d x$.

Both definitions presuppose the conditions of Theorem 1.12. These are essentially that $f(x)$ must be a real function of $x$, and that $k$ must be real and positive.

Adjusted forms of (1.4) and (1.5) are

$$
\begin{aligned}
2 C^{(m)} f & =\sum_{q=1}^{[(p-1) / 2]} \frac{K_{2 q}}{m^{2 q}}+\operatorname{Re} 4 \pi i \operatorname{Res}(2 \pi m)+2 C_{p}^{(m)}\left(L^{\prime}\right) f, \\
2 S^{(m)} f & =\sum_{q=0}^{[(p-2) / 2]} \frac{K_{2 q+1}}{m^{2 q+1}}+\operatorname{Im} 4 \pi i \operatorname{Res}(2 \pi m)+2 S_{p}^{(m)}\left(L^{\prime}\right) f .
\end{aligned}
$$

It is possible, under these definitions, to adjust a standard expansion to obtain another standard expansion. This may happen in (4.19) if $f(x)$ is periodic with period 1, in which case $K_{2 q}=0$ and the order of $C_{p}^{(m)}$ is $o\left(m^{-k}\right)$ for all $k$.

One may adjust the expansion for more than one pole by simply adding several separate residue terms instead of one.

Example. The following example, which was briefly mentioned in Section 3, illustrates the numerical effect of including the adjustment term in the F.C.A.E. This uses the function (1.8), namely

$$
f(x)=1 /\left(x^{2}-x+0.26\right) .
$$

Here, a simple pole, having residue $a_{-1}=\frac{1}{2} i \mu$, is located at

$$
c=\lambda+i \mu=0.5+(0.1) i
$$

and, in view of (4.16), we find the adjustment term to be

$$
\operatorname{Re}(2 \pi i \operatorname{Res}(2 \pi m))=(\pi / \mu)(-1)^{m} e^{-\pi m / 5} .
$$

The numbers below show the results obtained when a user attempts to calculate $C^{(6)} f$ by evaluating partial sums of the F.C.A.E. using on one hand the standard series (1.4) and on the other hand the adjusted series (4.19).

The correct result is

$$
C^{(6)} f \doteq 0.704033694
$$


TABLE 1

\begin{tabular}{rrcc}
\hline & $\begin{array}{c}\text { qth term in }(1.4) \\
\text { or in }(4.19)\end{array}$ & $\begin{array}{c}\text { qth partial sum } \\
\text { in }(1.4)\end{array}$ & $\begin{array}{c}\text { qth partial sum } \\
\text { in }(4.19)\end{array}$ \\
\hline 1 & $-2.081713996537-002$ & $-2.081713996537-002$ & $7.034491100931-001$ \\
2 & $6.240287755500-004$ & $-2.019311118929-002$ & $7.040731388726-001$ \\
3 & $-4.405942671467-005$ & $-2.023717061616-002$ & $7.040290794568-001$ \\
4 & $5.406335388194-006$ & $-2.023176428105-002$ & $7.040344857844-001$ \\
5 & $-9.689934463007-007$ & $-2.023273327446-002$ & $7.040335167781-001$ \\
6 & $2.189459081506-007$ & $-2.023251432867-002$ & $7.040337357321-001$ \\
7 & $-4.886759442277-008$ & $-2.023256319633-002$ & $7.040336868609-001$ \\
8 & $-1.259169531957-009$ & $-2.023256445536-002$ & $7.040336856153-001$ \\
9 & $2.215382693859-008$ & $-2.023254230153-002$ & $7.040337077575-001$ \\
10 & $-3.699575480714-008$ & $-2.023257929715-002$ & $7.040336707723-001$ \\
11 & $5.432548918994-008$ & $-2.023252497194-002$ & $7.040337250917-001$ \\
12 & $-7.975547956536-008$ & $-2.023260472750-002$ & $7.040336453356-001$ \\
13 & $1.182895543519-007$ & $-2.023248643789-002$ & $7.040337636252-001$ \\
14 & $-1.688494712958-007$ & $-2.023265528725-002$ & $7.040335947764-001$ \\
15 & $1.911763558659-007$ & $-2.023246411118-002$ & $7.040337859537-001$ \\
16 & $4.555025903741-008$ & $-2.023241856077-002$ & $7.040338314953-001$ \\
17 & $-1.615341149154-006$ & $-2.023403390252-002$ & $7.040322161629-001$ \\
18 & $9.184600203531-006$ & $-2.022484930232-002$ & $7.040414007613-001$ \\
19 & $-4.237003493705-005$ & $-2.026721933740-002$ & $7.039990307181-001$ \\
20 & $1.809352162352-004$ & $-2.008628412092-002$ & $7.041799659492-001$ \\
\hline
\end{tabular}

The elements and partial sums in two asymptotic expansions for the sixth $(m=6)$ cosine Fourier coefficient $C^{(6)} f$ of $f(x)=1 /\left(x^{2}-x+0.26\right)$. The numbers have an absolute accuracy of about $10^{-11}$.

and the closest approximation in the final column $(q=7)$ differs from this by only $6 \times 10^{-9}$. These results should be treated only as a qualitative illustration. The problem of placing some bound on the validity of such results remains and, unless the user carries out some analytical or numerical investigation of the remainder term, he has no way of knowing how good or bad any particular result might be.

5. The Euler-Maclaurin Expansion. One application of the Fourier coefficient asymptotic expansion is to derive the Euler-Maclaurin summation formula. This is described in Lyness and Ninham [3] for the case in which the singularities mentioned in Section 1 occur. It is described again in Lyness [4] using the same notation as used here in the regular case. Briefly, the displaced trapezoidal rule

$$
R^{[m, \alpha]} f=\frac{1}{m} \sum_{j=1}^{m} f\left(\frac{j+t_{\alpha}-1}{m}\right), \quad t_{\alpha}=(1+\alpha) / 2, \quad|\alpha|<1,
$$

may be expressed in terms of the Fourier coefficients of $f(x)$ by means of the Poisson summation formula

$$
R^{[m, \alpha]} f-I f=2 \sum_{r=1}^{\infty} \cos 2 \pi r t_{\alpha} C^{(r m)} f+2 \sum_{r=1}^{\infty} \sin 2 \pi r t_{\alpha} S^{(r m)} f .
$$

If we substitute expressions (1.4) and (1.5) for the Fourier coefficients, the summation over index $r$ may be carried out in terms of Bernoulli functions, leaving

$$
R^{[m, \alpha]} f-I f=\sum_{a=1}^{p-1} \frac{\bar{B}_{q}\left(t_{\alpha}\right)}{q !} \frac{f^{(\alpha-1)}(1)-f^{(\alpha-1)}(0)}{m^{\alpha}}+E_{p}^{[m, \alpha]} f,
$$


where the remainder term is given by

$$
E_{p}^{(m, \alpha)} f=2 \sum_{r=1}^{\infty} \cos 2 \pi r t_{\alpha} C_{p}^{(r m)} f+2 \sum_{r=1}^{\infty} \sin 2 \pi r t_{\alpha} S_{p}^{(r m)} f
$$

It is interesting to note the effect of using an adjusted form of the Fourier coefficient asymptotic expansion (4.19), (4.20) in (5.2) to obtain a correspondingly adjusted form of the Euler-Maclaurin expansion. This gives an additional term in (5.3), namely,

$$
\Delta^{[m, \alpha]}=\sum_{r=1}^{\infty} \cos 2 \pi r t_{\alpha} \operatorname{Re}(4 \pi i \operatorname{Res}(2 \pi m r))+\sum_{r=1}^{\infty} \sin 2 \pi r t_{\alpha} \operatorname{Im}(4 \pi i \operatorname{Res}(2 \pi m r))
$$

$$
=\operatorname{Re} \sum_{r=1}^{\infty} e^{-2 \pi i r t a} 4 \pi i \operatorname{Res}(2 \pi m r) .
$$

while the remainder term is altered by $\Delta^{[m, \alpha]}$ to

$$
E_{p}^{[m, \alpha]}\left(L^{\prime}\right)=2 \sum_{r=1}^{\infty} \cos 2 \pi r t_{\alpha} C_{p}^{(r m)}\left(L^{\prime}\right) f+2 \sum_{r=1}^{\infty} \sin 2 \pi r t_{\alpha} S_{p}^{(r m)}\left(L^{\prime}\right) f
$$

We now investigate the form of $\Delta^{[m, \alpha]}$ for the case in which $z=c$ is a simple pole.

THEOREM 5.7. When $z=c$ is a simple pole

$$
\Delta^{[m, \alpha]}=\operatorname{Re} 4 \pi i a_{-1} /\left(e^{2 \pi i t a} e^{-2 \pi i m c}-1\right) .
$$

Proof. Since $z=c$ is a simple pole we may use (4.16). Thus

$$
\operatorname{Res}(2 \pi m r)=a_{-1} e^{2 \pi i m r c} \text {. }
$$

The sum in (5.5) reduces to the sum of an infinite geometric progression; this may be summed to give (5.7).

The next theorem is less mundane:

THEOREM 5.9. If $z=c$ is a simple pole, then

$$
\Delta^{[m, \alpha]}=R^{[m, \alpha]} \phi,
$$

where

$$
\phi(x)=\operatorname{Re} \frac{4 \pi i a_{-1}}{e^{2 \pi i x} e^{-2 \pi i c}-1} .
$$

This may be proved in a direct manner. A proof which requires less manipulation is as follows. We may expand the denominator of (5.10) by the binomial theorem (since $\operatorname{Im} c>0$ ). This gives

$$
\phi(x)=\operatorname{Re} 2 \sum_{r=1}^{\infty} e^{-2 \pi i r x} e^{2 \pi i r c} 4 \pi i a_{-1} .
$$

Consequently, the Fourier coefficients of $\phi(x)$ are given by

$$
\begin{aligned}
& I \phi=0 ; \quad 2 C^{(r)} \phi=\operatorname{Re} 4 \pi i a_{-1} e^{2 \pi i r c}, \\
& 2 S^{(r)} \phi=-\operatorname{Re} 4 \pi a_{-1} e^{2 \pi i r c} .
\end{aligned}
$$

Applying the Poisson summation formula (5.2), we find 


$$
\begin{aligned}
R^{(m, \alpha)} \phi & =2 \sum_{r=1}^{\infty} \cos 2 \pi r t_{\alpha} C^{(r m)} \phi+2 \sum_{r=1}^{\infty} \sin 2 \pi r t_{\alpha} S^{(r m)} \phi \\
& =\operatorname{Re} \sum_{r=1}^{\infty} e^{-2 \pi i r t a} e^{2 \pi i r m c} 4 \pi i a_{-1} .
\end{aligned}
$$

In view of (5.8), this is identical to (5.5). This establishes Theorem 5.9.

Theorems 5.7 and 5.9 may be generalized for poles of order $\omega>1$. To this end, we introduce a set of functions as follows:

$$
\begin{gathered}
\psi_{0}(x, c)=\sum_{r=1}^{\infty} e^{2 \pi i r(c-x)} \\
\psi_{s}(x, c)=-\frac{\partial}{\partial x} \psi_{s-1}(x, c)=\sum_{r=1}^{\infty}(2 \pi i r)^{s} e^{2 \times i r(c-x)}, s=1,2, \cdots, \omega-1
\end{gathered}
$$

These may be evaluated in closed form. The first is the sum of a geometric progression, namely

$$
\psi_{0}(x, c)=1 /\left(e^{-2 \pi i(c-x)}-1\right) .
$$

Subsequent functions may be obtained by differentiation. For example,

$$
\psi_{1}(x, c)=-\frac{\partial}{\partial x} \psi_{0}(x, c)=\frac{2 \pi i e^{-2 \pi i(c-x)}}{\left(e^{-2 \pi i(c-x)}-1\right)^{2}} .
$$

It will be convenient to define the analytic continuation of these functions by

$$
\psi_{0}(z, c)=1 /\left(e^{2 \pi i(z-c)}-1\right), \quad \psi_{s}(z, c)=-\frac{\partial}{\partial z} \psi_{s-1}(z, c) .
$$

This definition is valid for all $z$, except at poles, which occur when $z-c$ is an integer.

If the pole at $z=c$ has order $\omega$, then

$$
\operatorname{Res}(k)=e^{i k c} \sum_{s=0}^{\omega-1} a_{-s-1}(i k)^{s} / s !
$$

Substitution of this value of Res $(k)$ into (5.5) gives

$$
\Delta^{[m, \alpha]}=\operatorname{Re} \sum_{r=1}^{\infty} \sum_{s=0}^{\omega-1} 4 \pi i a_{-s-1}(2 \pi i m r)^{s} e^{2 \pi i r(m c-t a)} / s ! .
$$

THEOREM 5.7'. If $z=c$ is a pole of order $\omega$, then

$$
\Delta^{[m, \alpha]}=\operatorname{Re} 4 \pi i \sum_{s=0}^{\omega-1} a_{-s-1} m^{s} \psi_{s}\left(t_{\alpha}, m c\right) / s ! .
$$

THEOREM 5.9'. If $z=c$ is a pole of order $\omega$, then

$$
\Delta^{[m, \alpha]}=R^{[m, \alpha]} \phi
$$

where

$$
\phi(x)=\operatorname{Re} 4 \pi i \sum_{s=0}^{\omega-1} a_{-s-1} \psi_{s}(x, c) / s ! .
$$

The proofs of Theorems $5.7^{\prime}$ and $5.9^{\prime}$ follow the same lines as those of Theorems 5.7 and 5.9. 
The function $\phi(x)$ has an analytic continuation

$$
\phi(z)=2 \pi i \sum_{s=0}^{\omega-1}\left(a_{-s-1} \psi_{s}(z, c)-\bar{a}_{-s-1} \psi_{s}^{*}(z, \bar{c})\right) / s !,
$$

where

$$
\psi_{0}^{*}(z, \bar{c})=1 /\left(e^{-2 \times i(z-\bar{c})}-1\right)
$$

and

$$
\psi_{s}^{*}(z, \bar{c})=(-1)^{s} \frac{\partial}{\partial z} \psi_{s-1}^{*}(z, \bar{c})
$$

We have already shown that $I \phi=0$ and by definition $\phi(z)$ is clearly periodic with period 1. We now prove that $\phi(z)$ has a pole at $z=c$ of precisely the same nature as the pole of $f(z)$.

THEOREM 5.27. $f(z)-\phi(z)$ is regular in a neighborhood of $z=c$.

Proof. In view of $(5.18), \psi_{0}(z, c)$ has a pole of order 1 . Thus, $\psi_{0}(z, c)$ may be expanded in the form

$$
\psi_{0}(z, c)=\frac{1}{2 \pi i(z-c)}+\sum_{r=0}^{\infty} \beta_{r}(z-c)^{r} .
$$

Consequently,

$$
\psi_{s}(z, c)=(-1)^{s} \frac{\partial^{s}}{\partial z^{s}} \psi_{0}(z, c)=\frac{s !}{(2 \pi i)(z-c)^{8+1}}+\sum \beta_{r}^{\prime}(z-c)^{r} .
$$

Substituting this into (5.24), we find

$$
\phi(z)=\sum_{s=0}^{\omega-1} a_{-s-1}(z-c)^{-s-1}-2 \pi i \sum_{s=0}^{\omega-1} \bar{a}_{-s-1} \psi_{s}^{*}(z, \bar{c}) / s !+\sum \beta_{r}^{\prime \prime}(z-c)^{r} .
$$

The first term on the right coincides with the 'infinite part' of $f(z)$ given by (4.12). The second term has poles only at the poles of $\psi_{s}^{*}(z, \bar{c})$. These occur where $z-\bar{c}$ is an integer. Since $\mu \neq 0$, the second term is regular at $z=c$, as is the third. Consequently, $\phi(z)-f(z)$ is regular at $z=c$, which establishes the theorem.

The adjusted Euler-Maclaurin summation formula takes the form

$$
R^{[m, \alpha]} f-I f=\sum_{a=1}^{p-1} \frac{\bar{B}_{q}\left(t_{\alpha}\right)}{q !} \frac{f^{(q-1)}(1)-f^{(\alpha-1)}(0)}{m^{q}}+\Delta^{[m, \alpha]}+E_{p}^{[m, \alpha]}\left(L^{\prime}\right) f .
$$

The term $\Delta^{[m, \alpha]}$ may be evaluated directly using (5.7). It is interpreted using (5.9) as $R^{[m, \alpha]} \phi$. By the previous theorem, $\phi(z)-f(z)$ is regular at $z=c$. Thus the introduction of $\Delta^{[m, \alpha]}$ corresponds precisely to the process of subtracting out the singularity. For example, the adjusted formula (5.30) may be written in the form

$$
R^{[m, \alpha]} \chi-I \chi=\sum_{a=1}^{p-1} \frac{\bar{B}_{\alpha}\left(t_{\alpha}\right)}{q !} \frac{\chi^{(q-1)}(1)-\chi^{(a-1)}(0)}{m^{\alpha}}+E_{p}^{[m, \alpha]}\left(L^{\prime}\right) f,
$$

where $\chi(x)=f(x)-\phi(x)$.

Suppose, for example, that Gregory's quadrature rule is used. After evaluating $R^{[m, \alpha]} f$ and the approximations, based on finite differences, to $f^{(a-1)}(1)$ and $f^{(a-1)}(0)$, it may be realized that a nearby pole is important. Rather than subtracting out the 
singularity and recommencing the calculation with $\chi(x)$, all that is necessary to obtain an identical result is to subtract the number $\Delta^{[m, \alpha]}$ given by (5.7). The author need hardly add that he does not recommend such a haphazard approach to numerical quadrature, but such a situation does illustrate the relation between the adjusted formula (5.30) and a process for subtracting out the singularity.

6. Applications to Numerical Quadrature. Subtracting out a pair of complex conjugate poles would not normally be accomplished using $\phi(x)$ as a subtraction function. In this section, we discuss the conventional approach to the problem and compare the two methods.

The first thing that has to be done is to determine the location and nature of the nearby singularities. This in itself can be quite tedious. We suppose that this has been done and we have found simple poles at $c=\lambda+i \mu$ and at $\bar{c}$. We now can express $f(x)$ in the form

$$
f(x)=\frac{\theta(x)}{(x-c)(x-\bar{c})},
$$

where the value of $\theta(c)$ is known, i.e.,

$$
\theta(c)=A+i B, \quad c=\lambda+i \mu .
$$

A direct approach would be to choose a function $h(x)$ such that $h(z)-f(z)$ is regular at $z=c$ and at $z=\bar{c}$. The usual choice is

$$
h(x)=\operatorname{Re} \frac{\theta(c)}{i \mu(x-c)}=\frac{1}{\mu}\left\{\frac{\mu A+(x-\lambda) B}{(x-\lambda)^{2}+\mu^{2}}\right\} .
$$

An alternative choice, derived in the previous section, is

$$
\begin{aligned}
\phi(x) & =\frac{2 \pi}{\mu} \operatorname{Re} \frac{\theta(c)}{e^{2 \pi i(x-c)}-1} \\
& =\frac{2 \pi}{\mu}\left\{\frac{A\left[e^{2 \pi \mu} \cos 2 \pi(\lambda-x)-1\right]-B e^{2 \pi \mu} \sin 2 \pi(\lambda-x)}{1-2 e^{2 \pi \mu} \cos 2 \pi(\lambda-x)+\left(e^{2 \pi \mu}\right)^{2}}\right\} .
\end{aligned}
$$

In general, one evaluates $I h$ or $I \phi$ analytically and applies the quadrature rule to the function $f(x)-h(x)$ or $f(x)-\phi(x)$. So far as the analytic evaluation of $I h$ is concerned, although this is straightforward, it is not quite as easy as the result $I \phi=0$. However, when one comes to apply the quadrature rule, in general the function $f(x)-h(x)$ is usually easier to evaluate at an abscissa than the function $f(x)-\phi(x)$. Without stating the form of $f(x)$, one cannot say for certain that this would be the case, particularly as it is normal to simplify the expression $f(x)-h(x)$ or $f(x)-\phi(x)$ before applying the quadrature rule. However, to evaluate $h(x)$ one is, in general, committed to evaluating $(x-\lambda)$ at each abscissa. To evaluate $\phi(x)$, both $\cos 2 \pi(x-\lambda)$ and $\sin 2 \pi(x-\lambda)$ in addition have to be evaluated at each abscissa.

A second inconvenience is that the function $\phi(z)$ has poles at all points $z=n+$ $\lambda \pm i \mu, n=0, \pm 1, \pm 2$. Thus, while $f(x)-\phi(x)$ has no poles at $\lambda \pm i \mu$, it does have poles at $\lambda \pm 1 \pm i \mu$. If $\lambda$ is close to either 1 or 0 , these additional poles may cause inconvenience in the numerical quadrature process.

While in general the use of $\phi(x)$ rather than $h(x)$ as a subtraction function has 
TABLE 2

Elements of two Romberg T-tables based on (6.8) and (6.9), respectively.

\begin{tabular}{|c|c|c|c|c|c|c|c|}
\hline$i m_{i}$ & $T_{0}$ & & $T_{1}^{i-1}$ & $T_{2^{i-2}}$ & $T_{3}^{i-3}$ & $T_{4}^{i-4}$ & $T_{6}^{i-b}$ \\
\hline $\begin{array}{ll}0 & 1 \\
1 & 2 \\
2 & 3 \\
3 & 4 \\
4 & 6 \\
5 & 8\end{array}$ & $\begin{array}{r}8.5998 \\
8.9031 \\
-3.1087 \\
1.9623 \\
5.1533 \\
1.3721\end{array}$ & $\begin{array}{l}-001 \\
-001 \\
-001 \\
-001 \\
-002 \\
-002\end{array}$ & $\begin{array}{r}1.4737-000 \\
-1.2718+000 \\
8.4821-001 \\
-6.4226-002 \\
-3.4895-002\end{array}$ & $\begin{array}{r}-1.6150+000 \\
1.5549+000 \\
-3.6837-001 \\
-2.5118-002\end{array}$ & $\begin{array}{r}1.7662+000 \\
-6.0878-001 \\
3.1051-002\end{array}$ & $\begin{array}{r}-6.7664-001 \\
7.3707-002\end{array}$ & $8.5617-002$ \\
\hline $\begin{array}{ll}0 & 1 \\
1 & 2 \\
2 & 3 \\
3 & 4 \\
4 & 6 \\
5 & 8\end{array}$ & $\begin{array}{l}-6.4190 \\
-1.9726 \\
-9.3333 \\
-5.3907 \\
-2.4470 \\
-1.3875\end{array}$ & $\begin{array}{l}-002 \\
-002 \\
-003 \\
-003 \\
-003 \\
-003\end{array}$ & $\begin{array}{l}-4.9050-003 \\
-1.0189-003 \\
-3.2173-004 \\
-9.2088-005 \\
-2.5145-005\end{array}$ & $\begin{array}{l}-5.3314-004 \\
-8.9340-005 \\
-1.5540-005 \\
-2.8310-006\end{array}$ & $\begin{array}{l}-5.9753-005 \\
-6.3153-006 \\
-7.5130-007\end{array}$ & $\begin{array}{l}-4.7885-006 \\
-3.8037-007\end{array}$ & $-3.1041-007$ \\
\hline
\end{tabular}

little to recommend it, there are two special interrelated cases in which it might be useful.

Firstly, for a general function, one might use the trapezoidal rule, or some rule closely related to the trapezoidal rule such as Simpson's rule or Romberg integration. In this case the first objection to using $\phi(x)$ related to ease of calculation disappears. In fact, one finds that, from a computational point of view, $\phi(x)$ is easier to use than $h(x)$. This is because

$$
\begin{aligned}
R^{[m, 1]}(f-\phi) & =R^{[m, 1]} f-R^{[m, 1]} \phi \\
& =R^{[m, 1]} f-\operatorname{Re} \frac{2 \pi}{\mu} \frac{\theta(c)}{e^{-2 \pi i m c}-1} .
\end{aligned}
$$

Instead of evaluating the function $f(x)-\phi(x)$ at each of the $m+1$ abscissas, one adjusts the sum of $m+1$ function values of $f(x)$ by a term whose evaluation is equivalent to a single function evaluation of $\phi(x)$. In fact, if a regular sequence of values of $m$ is used, only one or two exponentials are required for all values of $m$ for this additional term. No such simplification is known to the author for the evaluation of $R^{[m, 1]}(f-h)$, which requires $m+1$ function values of $f(x)-h(x)$. However, the second objection mentioned above, that relating to additional induced poles, is still valid, and this procedure would be useful if the pole is significantly closer to the real axis than to either of the lines $\operatorname{Re}(z)=0$ or $\operatorname{Re}(z)=1$.

As an example, we consider the evaluation of If $=\int_{0}^{1} f(x) d x$, where

$$
f(x)=1 /\left(x^{2}-x+0.26\right)
$$

using Romberg integration with mesh ratios

$$
\left\{m_{i}\right\}=\{1,2,3,4,6,8\}, \quad i=0,1,2, \cdots, 5 .
$$

In the first half of Table 2, the first column of the $T$-table is based on

$$
T_{0}^{i}=R^{\left[m_{i}, 1\right]} f \text {. }
$$

In the second half of Table 2, the first column is based on

$$
T_{0}^{i}=R^{[m i, 1]} f-\Delta^{\left[m_{i}, 1\right]} .
$$

Here the adjustment term, given by (5.7) is 


$$
\Delta^{[m, 1]}=20 \pi /\left((-1)^{m} e^{\pi m / 5}-1\right) .
$$

In both tables, the rest of the $T$-table is calculated from the first column in the conventional manner. In both cases, we have listed, in place of $T_{i}^{i}$, the signed relative error

$$
E_{i}^{i}=T_{j}^{i} / \text { If }-1 \text {. }
$$

The second special case is that in which $f(x)$ is analytic and has period 1 . In this case, it is natural to use a quadrature rule which takes advantage of the periodicity of the function and so one would be inclined to use a subtraction function which has the same property, i.e., $\phi(x)$ and not $h(x)$.

We may be more definite than this. If $f(x)$ is analytic and periodic, then $f(z)$ has poles of a similar nature at $z=n+\lambda \pm i \mu$, and $\phi(z)$ subtracts out all these poles. The second objection is no longer valid; rather, the usually inconvenient property becomes an advantage. In addition, the evaluation of a function $f(x)$ of this nature almost certainly involves the evaluation of $\cos 2 \pi x$ or $\sin 2 \pi x$. By a proper arrangement of the interval of integration, the same values of $\sin 2 \pi x$ and $\cos 2 \pi x$ may be used in the evaluations of both $f(x)$ and $\phi(x)$. Thus, the first objection need not apply. However, in such cases it is conventional to use the trapezoidal rule, and as indicated above, $\phi(x)$ need not be calculated at each abscissa.

Finally, we mention that this subtraction function can be extremely convenient when applied to the MIPS method, Lyness [4], for calculating Fourier coefficients. This application is described in a preceding paper in this issue.

APPENDIX. F.C.A.E. for Logarithmic-Algebraic Singularities. An asymptotic expansion similar to the one stated in Theorem 1.12 is valid in the case in which $f(x)$ has an algebraic logarithmic singularity at a terminal point. We treat the function

$$
\begin{aligned}
f(z) & =\ln (x-a)(x-a)^{\alpha}(b-x)^{\beta} h(x), \quad \alpha>0, \beta>-1, \\
& =\ln (x-a)(x-a)^{\alpha} \psi(x) .
\end{aligned}
$$

The derivation of the expansion is structurally similar to that given in Section 2. In this Appendix, we note briefly the difference in detail.

The coefficients that occur involve the digamma function $\psi(x)$ defined by

$$
\begin{aligned}
\Gamma(z) \psi(z) & =\Gamma^{\prime}(z)=\frac{d}{d z} \int_{0}^{\infty} e^{-t} t^{z-1} d t \\
& =\int_{0}^{\infty} e^{-t} t^{z-1} \ln t d t .
\end{aligned}
$$

We again expand the regular part of $f(x)$ at $x=a$ as a Taylor series to give

$$
g(z)=\ln (z-a)(z-a)^{\alpha} \sum_{a=0}^{p-1} \frac{\psi^{(a)}(a)(z-a)^{a-1}}{(q-1) !} .
$$

The terms in the expansion, as in Lemma 2.4, come from the following evaluation of an integral:

$$
\int_{a}^{a+i \infty} g(z) e^{i k z} d z=\sum_{a=0}^{p-1} \frac{\psi^{(\alpha)}(a)}{(q-1) !} e^{i \pi \alpha / 2} e^{i k a} i^{a+1} \int_{0}^{\infty}\left(\ln y+\frac{i \pi}{2}\right) y^{a+\alpha} e^{-k y} d y .
$$

The term $(\ln y+i \pi / 2)$ is the value of $\ln (z-a)$ at $z=a+i y$. 
The final integral may be manipulated using (A.1) to give

$$
\begin{array}{rl}
\int_{0}^{\infty}\left(\ln y+\frac{i \pi}{2}\right) y^{a+\alpha} e^{-k y} & d y=\frac{1}{k^{a+\alpha+1}} \int_{0}^{\infty}\left(\ln t-\ln k+\frac{i \pi}{2}\right) t^{a+\alpha} e^{-t} d t \\
= & \frac{\Gamma(q+\alpha+1)}{k^{a+\alpha+1}}\left[\psi(q+\alpha+1)-\ln k+\frac{i \pi}{2}\right] .
\end{array}
$$

The part of the expansion involving derivatives of $\psi(x)$ at $x=a$ contains both terms in $k^{-a-a-1}$ and terms in $\ln k k^{-a-\alpha-1}$. These are

$$
\begin{aligned}
& \int_{a}^{a+i \infty} g(z) e^{i k z} d z \\
= & e^{i \pi \alpha / 2} e^{i k \alpha} \sum_{a=0}^{p-1} \frac{\psi^{(a)}(a)}{q !} \frac{i^{a+1}}{k^{\alpha+a+1}} \Gamma(\alpha+q+1)[\psi(q+\alpha+1)-\ln k+i \pi / 2] .
\end{aligned}
$$

The other part of the expansion is formally the same as in (1.12); however, in this case, $\phi(x)=\ln (x-a)(x-a)^{\alpha} h(x)$.

The verification of this result depends on establishing lemmas corresponding to Lemmas 2.1, 2.2, and 2.3. Lemma 2.1 is unaltered. Lemmas 2.2 and 2.3 have to be adjusted. The adjustment involves establishing the order of a function analogous to the incomplete gamma function (2.13). This function is

$$
\omega(\beta+1, x)=\int_{x}^{\infty} t^{\beta} e^{-t} \ln t d t \sim O\left(x^{\beta} e^{-x} \ln x\right), \quad x \rightarrow \infty .
$$

With this result, the derivation of the analogues of these lemmas may proceed along the same lines as in Section 2. The results are

$$
I_{2} \sim O\left(k^{-\alpha-p-1} \ln k\right), \quad I_{3} \sim O\left(k^{-1} \ln k e^{-k L}\right) .
$$

Thus, the order of the remainder term is established and coincides as before with the order of the first omitted term in the expansion.

Argonne National Laboratory

9700 South Cass Avenue

Argonne, Illinois 60439

1. A. ERdÉLYI, "Asymptotic representations of Fourier integrals and the method of stationary phase," J. Soc. Indust. Appl. Math., v. 3, 1955, pp. 17-27. MR 17, 29.

2. M. J. LighthiLL, Introduction to Fourier Analysis and Generalized Functions, Cambridge Univ. Press, New York, 1958; 1960. MR 19, 1066; MR 22 \#888.

3. J. N. LyNESS \& B. W. NiNHAM, "Numerical quadrature and asymptotic expansions," Math. Comp., v. 21, 1967, pp. 162-178. MR 37 \#1081.

4. J. N. LYNESS, "The calculation of Fourier coefficients by the Möbius inversion of the Poisson summation formula. I," Math. Comp., v. 24, 1970, pp. 101-135. 